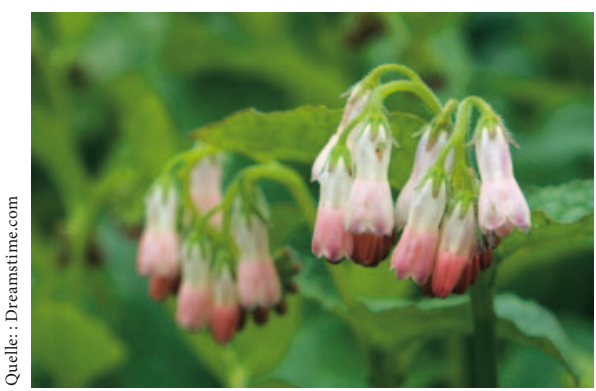

«Im Medizinstudium spielt die Phytotherapie in der Schweiz praktisch keine Rolle. Nur das Institut für Naturheilkunde der Universität Zürich unterrichtet diese Disziplin», sagt Prof. Dr. Beat Meier, Dozent für Phytopharmazie an der Zürcher Hochschule für angewandte Wissenschaften (ZHAW) in Wädenswil und gleichzeitig Geschäftsstellenleiter der SMGP. Trotzdem ist der Trend hin zu den verschiedenen Verfahren der Naturheilkunde ungebremst, und $\mathrm{Pa}$ tientinnen und Patienten verlangen zunehmend nach der Behandlung mit pflanzlichen Arzneimitteln. Ärztinnen und Ärzte werden immer stärker mit dem Wunsch der Patienten nach einem phytotherapeutischen Ansatz konfrontiert. «Viele entschliessen sich einige Zeit nach dem Studium, eine Weiterbildung in der Phytotherapie in Angriff zu nehmen. Wir würden uns jedoch auch wünschen, dass vermehrt jüngere Ärztinnen und Ärzte während ihrer Assistenzzeit einsteigen», sagt Meier. Im Gegensatz zur Anzahl der zugelassenen pflanzlichen Arzneimittel ist die Kompetenz vieler Ärztinnen und Ärzte in der Schweiz im Umgang mit den vielfältigen Möglichkeiten der qualifizierten Anwendung der Phytotherapie gering.

\section{Bedarf an Weiterbildung}

Die Fort- und Weiterbildung für Ärzte in der Phytotherapie wird hauptsächlich von der SMGP organisiert. In Zusammenarbeit mit der ZHAW und

\title{
Pflanzenheilkunde ist etwas für erfahrene Ärzte
}

Wer sich als Medizinerin oder Mediziner einen Rucksack an Wissen in der Phytotherapie zulegen will, sollte Berufserfahrung und eine Menge Zeit mitbringen. Die Weiterbildung der Schweizerischen Medizinischen Gesellschaft für Phytotherapie (SMGP) dauert drei Jahre.

dem Lehrstuhl für Naturheilkunde an der Universität Zürich werden eine Weiterbildung als Kurs über drei Jahre, aber auch Einzelveranstaltungen zur Fortbildung angeboten. Der Abschluss ist der Ausweis UNION/ SMGP Phytotherapie. Apotheker können den Antrag auf Vergabe des Fähigkeitsausweises FPH in Phytotherapie bei der FPH Offizin stellen.

«Die Ausbildung ist anspruchsvoll», sagt Meier. Der dreijährige Ausbildungszyklus besteht aus drei Pflichtmodulen, einem Zusatzmodul und einer Abschlussarbeit. Im ersten Modul geht es darum, Kenntnisse über die Biologie und Wirkungsweise der wichtigsten Arzneipflanzen zu vermitteln. Die Kursteilnehmer lernen, sie in der Natur zu erkennen, und auch die Prinzipien der Verarbeitung. Das zweite Modul liefert Kenntnisse über die Durchführung von klinischen Studien und von klinischer Forschung im Allgemeinen. Im dritten, dem stundenmässig umfangreichsten Modul geht es schliesslich um die Anwendung der Phytotherapie innerhalb der verschiedenen Indikationsgebiete.

\section{Botanik ist wichtig}

Auf den Exkursionen und in den Tagungen im 4. Modul werden die feldbotanischen Fähigkeiten vertieft und die Teilnehmer über die neuesten Erkenntnisse der Anwendung der pflanzlichen Arzneimittel orientiert. Am Ende der Ausbildung steht eine Abschlussarbeit.
Die Nachfrage nach den Weiterbildungen, die unter der Ägide der SMGP angeboten werden, ist in den letzten Jahren in etwa gleich geblieben. «Die Anzahl der Teilnehmer an den Kursen schwankt zwischen 30 und 40 Leuten», sagt SMGP-Präsident Beat Meier. Stets wachsend ist allerdings das Interesse an der Phytotherapie: Die Mitgliederzahl der SMGP steigt seit Jahren kontinuierlich an. (rfi)

\section{Hüterin und Förderin der Pflanzenheilkunde}

Die SMGP wurde 1988 gegründet und hat sich zum Ziel gemacht, die Phytotherapie im Schweizer Gesundheitswesen zu verbreiten und den Ausbildungsstand der Ärzteschaft zu verbessern. Heute hat die SMGP etwa 650 Mitglieder und die Last der Arbeit ruht auf den Schultern von knapp 50 neben- und ehrenamtlich tätigen Mitarbeitenden, die sich um die Angebote der Gesellschaft kümmern. Jedes Jahr veranstaltet die SMGP Ausund Weiterbildungskurse. In Lausanne wird die Fort- und Weiterbildung in komplettem Umfang auch auf Französisch angeboten. Auf Exkursionen, die dreimal pro Jahr stattfinden, werden verschiedene botanisch interessante Gebiete der Schweiz besucht. Auf der Internetseite (www.smgp.ch) werden Neuigkeiten dokumentiert und der Bericht von der Jahrestagung veröffentlicht, die der Höhepunkt im Jahresprogramm ist. Die 25. Ausgabe findet am 25. November 2010 in Baden statt.

\section{KARGER}

Fax +49761 4520714 